\title{
The Spinning Motions of All Fermions and Bosons as Implied by Pauli Matrices Containing Complex Conjugates in a Combined Spacetime Four-Manifold
}

\author{
Gregory L. Light ${ }^{1}$ \\ ${ }^{1}$ Department of Management, Providence College, 1 Cunningham Square, Providence, Rhode Island, USA \\ Correspondence: Gregory L. Light, Department of Management, Providence College, 1 Cunningham Square, \\ Providence, Rhode Island 02918, USA. Tel: 1-401-865-2662. E-mail: glight@providence.edu
}

Received: June 5, 2013 Accepted: June 25, 2013 Online Published: July 9, 2013

doi:10.5539/apr.v5n4p37 URL: http://dx.doi.org/10.5539/apr.v5n4p37

\begin{abstract}
By substituting $z=a+b i$ and $1 / z=a-b i$ for $i$ and $-i$ into one of Pauli matrices and then casting $(x, y)=(0, a+b i)$ as $(x, y, z)=(0, a, b)$ and $(x, y)=(a-b i, 0)$ as $(x, y, z)=(a,-b, 0)$ by the geometry of our previously formulated combined spacetime 4-manifold $=\{(t+t i, x+y i, y+z i, z+x i)\}$, this paper generalizes the Dirac equation for a free electron into an equation that gives the motion $(t, x(t), y(t), z(t))$ for any free fermion or boson to be a uniform circular flow around two semi-circles connected with each other by an angle equal to 0, 30, 60, 90, or 180 degrees depending on the electric charge possessed by the particle. Even purely algebraically, any fermion or boson must correspond to a number on the complex unit circle, since the Dirac equation admits a Pauli matrix of $z$ with modulus equal to one but not necessarily equal to $i$ and all energies in free space must satisfy this Dirac equation as derived from the universally true equation of "energy-squared minus pc-squared equal to rest-energy-squared."
\end{abstract}

Keywords: neutrino Majorana parity, generalized Pauli matrices, scattering mixing angles, quark plasma, Dirac spinors

\section{Introduction}

This paper extends our previous analysis of a free electron-wave (Light, 2013), where we showed its motion being that around two perpendicularly connected semi-circles by casting Pauli matrices in the Dirac equation in our proposed combined spacetime 4-manifold as

$$
\begin{gathered}
\sigma_{1} \equiv\left(\begin{array}{ll}
0 & 1 \\
1 & 0
\end{array}\right)_{(x, y)} \equiv\left(\begin{array}{ll}
0 & 1 \\
1 & 0 \\
0 & 0
\end{array}\right)_{(x, y, z)}, \\
\sigma_{2} \equiv\left(\begin{array}{cc}
1 & 0 \\
0 & -1
\end{array}\right)_{(x, z)} \equiv\left(\begin{array}{cc}
1 & 0 \\
0 & 0 \\
0 & -1
\end{array}\right)_{(x, y, z)},
\end{gathered}
$$

and

$$
\sigma_{3} \equiv\left(\begin{array}{cc}
0 & -i \\
i & 0
\end{array}\right)_{(x, y)} \equiv\left(\begin{array}{cc}
0 & 0 \\
0 & -1 \\
1 & 0
\end{array}\right)_{(x, y, z)}
$$

Here we extend $\sigma_{3}$ to 


$$
\tilde{\sigma}_{3}:=\left(\begin{array}{cc}
0 & a-b i \\
a+b i & 0
\end{array}\right)_{(x, y)} \equiv\left(\begin{array}{cc}
0 & a \\
a & -b \\
b & 0
\end{array}\right)_{(x, y, z)},
$$

$\forall a, b$ such that $a^{2}+b^{2}=1$, so that

$$
\tilde{\sigma}_{3}^{2}=I
$$

and the Dirac equation is still satisfied (to date no generalizations of Pauli matrices similar to ours in the context of the above Equation (1.5) existing in the literature, for generalizations of Pauli matrices into higher Clifford dimensions, see, e.g, Le'vay, 2008). Since Dirac deduced his equation strictly from

$$
E^{2}-(p c)^{2}=\left(m_{0} c^{2}\right)^{2}
$$

which governs any object with energy, we will use

$$
\begin{aligned}
& \frac{\sqrt{3}}{2} \pm \frac{1}{2} i \text { to describe quarks }\{d, s, b\}, \\
& \frac{1}{2} \pm \frac{\sqrt{3}}{2} i \text { to describe quarks }\{u, c, t\}
\end{aligned}
$$

and the degenerate case

$$
1 \pm 0 i \text { to describe neutrinos (where } \tilde{\sigma}_{3}=\sigma_{1} \text { ). }
$$

It might be worth repeating our overall logic here: Any particle with energy must obey Equation (1.6), $E^{2}-(p c)^{2}=\left(m_{0} c^{2}\right)^{2}$, which implies the Dirac equation (for a free electron), but the Dirac equation admits the generalized Pauli matrix $\tilde{\sigma}_{3}$ with $a+b i \neq i, a^{2}+b^{2}=1$; therefore, any non-electron particle must be in correspondence with a specific complex number $a_{0}+b_{0} i$ of modulus 1 in the Pauli matrix $\tilde{\sigma}_{3}$, whether with or without our proposed spacetime geometry of a combined 4-manifold. With our combined 4-manifold $\boldsymbol{M}^{[3]}:=$ $\{(t+t i, x+y i, y+z i, z+x i)\}$, however, the Dirac equation yields an exact 4-dimensional motion picture of any free particle-wave via $\tilde{\sigma}_{3}$. This picture shows a uniform circular motion around two semi-circles connected with an acute angle $=0,30,60$, and 90 degrees respectively for the three generations of neutrinos, down quarks, up quarks, and electrons. This geometry explains in particular: (1) the above particles have rest masses because their wave motions must stop at the intersections of the two semi-circles for conservation of angular momenta, and (2) neutrinos have only one common spin direction because the two semi-circles coincide. As a motivation, we consider proton, made up of $\{u, u, d\}$. In our analysis, these three particle-waves coincide as one common ball known as proton for the reason that the quantum probability density for any particle of $\{u, u, d\}$ to appear is proportional to $r^{-4}$ to the ball center $r=0$ by the property of the electric field (see Equation (2.4) below), so that $\{u, u, d\}$ effectively present themselves as one point. As a consequence, their associated mini black holes are connected. The boundary of any black hole has proper time $\Delta t=0$ so that $\Delta \mathrm{x}=c \Delta t=0$ and the entire boundary collapses topologically into one point. This quotient-space topology then reduces the three mini black holes to one and as a result $\{u, u, d\}$ bind as a single proton.

In the next Section 2 we will formulate our ideas mathematically and apply our geometry to some baryons and mesons for an illustration. Then in Section 3 we will conclude with a summary remark.

\section{Analysis}

Dirac applied Clifford algebra to the equation

$$
E^{2}-(p c)^{2}=\left(m_{0} c^{2}\right)^{2}
$$

and derived his equation

$$
\left(P_{t}+c \sigma_{j} P_{(x, y, z)}\right)\left(\psi_{A}, \psi_{B}\right)=m_{0} c^{2}\left(\psi_{A}, \psi_{B}\right)
$$


where

$$
P_{t}:=i \hbar \frac{\partial}{\partial t} \quad \text { and } \quad P_{(x, y, z)}:=-i \hbar \nabla,
$$

Therein $\mathrm{i} \nabla \equiv \mathrm{a} 90$-degree rotation of the gradient vector to arrive at the tangent vector $=$ the direction of the momentum vector; since perpendicular to a gradient is a plane, this 90 -degree rotation as represented by $i$ is determined by the momentum vector from the equation of motion prescribed in advance.

Previously we postulated the (position) wavefunction for a free electron (Light, 2013, Equation (2.8), which connected quantum mechanics to the classical electromagnetic field theory) to be:

$$
\begin{aligned}
& \psi(t, x, y, z)=z_{0} e^{-i \cdot \frac{\omega}{2} t}\|E(x, y, z)\|_{\mathbb{C}^{3}}=z_{0} e^{-i \cdot \frac{\omega}{2} t} \cdot \frac{q}{4 \pi \varepsilon_{o}\left(x^{2}+y^{2}+z^{2}\right)}, \\
& \text { where } \omega \equiv k c \equiv 2 \pi v \equiv k \lambda v,
\end{aligned}
$$

and based on which we derived our solution to the Dirac equation for the motion of a free electron-wave to be (for solutions to Dirac equations, without the geometry of our proposed combined spacetime 4-manifold, cf. e.g., Taub, 1949; Barut \& Bracken, 1981; and the closest to our description, Rafanelli \& Schiller, 1964):

$$
\begin{aligned}
& \psi_{A}(t) \equiv \psi\left(t, x_{A}(t), y_{A}(t), z_{A}(t)\right) \equiv \psi\left(t, \Lambda_{A}(t)\right), \\
& \psi_{B}(t) \equiv \psi\left(t, x_{B}(t), y_{B}(t), z_{B}(t)\right) \equiv \psi\left(t, \Lambda_{B}(t)\right),
\end{aligned}
$$

with

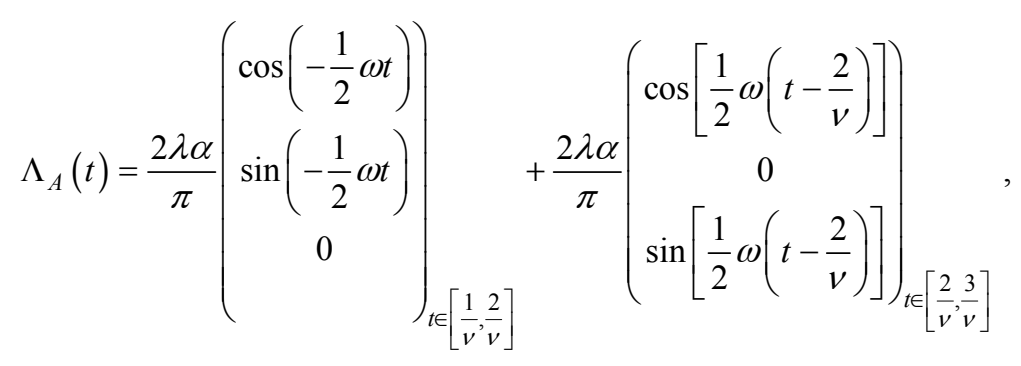

and

$$
\Lambda_{B}(t)=\frac{2 \lambda \alpha}{\pi}\left(\begin{array}{c}
\cos \left(-\frac{\pi}{2}-\frac{1}{2} \omega t\right) \\
0 \\
\sin \left(-\frac{\pi}{2}-\frac{1}{2} \omega t\right)
\end{array}\right)_{t \in\left[\frac{1}{v}, \frac{2}{v}\right]}+\frac{2 \lambda \alpha}{\pi}\left(\begin{array}{c}
0 \\
\cos \left[-\frac{\pi}{2}-\frac{1}{2} \omega\left(t-\frac{2}{v}\right)\right] \\
\sin \left[-\frac{\pi}{2}-\frac{1}{2} \omega\left(t-\frac{2}{v}\right)\right]
\end{array}\right)_{t \in\left[\frac{2}{v}, \frac{3}{v}\right]},
$$

with the value of $\alpha$ to be revealed later in Equation (2.17).

To facilitate our analysis and presentation, hereafter we shall adopt the following notations:

$$
\begin{aligned}
E & \equiv \frac{2 \lambda \alpha}{\pi}(1,0,0), \quad W \equiv \frac{2 \lambda \alpha}{\pi}(-1,0,0), \\
N & \equiv \frac{2 \lambda \alpha}{\pi}(0,1,0), \quad S \equiv \frac{2 \lambda \alpha}{\pi}(0,-1,0), \\
T & \equiv \frac{2 \lambda \alpha}{\pi}(0,0,1), \quad B \equiv \frac{2 \lambda \alpha}{\pi}(0,0,-1) .
\end{aligned}
$$

Then we have 


$$
\Lambda_{A}\left(t=\frac{1}{v}, \frac{1.5}{v}, \frac{2}{v}\right)=(W, N, E)
$$

and

$$
\begin{aligned}
& \left.\nabla \psi_{A}\right|_{t=\frac{1}{v}, \frac{1.5}{v}, \frac{2}{v}}=\left.\frac{-2 \psi_{A}}{x^{2}+y^{2}+z^{2}} \cdot(x, y, z)\right|_{t=\frac{1}{v}, \frac{1.5}{v}, \frac{2}{v}} \\
& =-2 \psi_{A} \cdot \frac{\pi}{2 \lambda \alpha} \cdot\{(-1,0,0) ;(0,1,0) ;(1,0,0) \mid \text { respectively }\} \\
& =\frac{\pi}{\lambda \alpha} \psi_{A} \cdot\{(1,0,0) ;(0,-1,0) ;(-1,0,0) \mid \text { respectively }\} .
\end{aligned}
$$

Accordingly a 90-degree rotation leads to

$$
\begin{aligned}
& \left.i \nabla \psi_{A}\right|_{t=\frac{1}{v}, \frac{1.5}{v}, \frac{2}{v}}=\left.\frac{k}{2 \alpha} \cdot \psi_{A}\right|_{t=\frac{1}{v}, \frac{1.5}{v}, \frac{2}{v}} \cdot\{(0,1,0) ;(1,0,0) ;(0,0,1) \mid \text { respectively }\}, \\
& \text { where } \frac{k}{2 \alpha} \equiv \frac{\pi}{\lambda \alpha} .
\end{aligned}
$$

Analogously we have

$$
\begin{aligned}
& \Lambda_{B}\left(t=\frac{1}{v}, \frac{1.5}{v}, \frac{2}{v}\right)=(T, E, B), \\
& \left.\nabla \psi_{B}\right|_{t=\frac{1}{v}, \frac{1.5}{v}, \frac{2}{v}}=\frac{\pi}{\lambda \alpha} \psi_{B} \cdot\{(0,0,-1) ;(-1,0,0) ;(0,0,1) \mid \text { respectively }\}, \text { and } \\
& \left.i \nabla \psi_{B}\right|_{t=\frac{1}{v}, \frac{1.5}{v}, \frac{2}{v}}=\left.\frac{k}{2 \alpha} \cdot \psi_{B}\right|_{t=\frac{1}{v}, \frac{1.5}{v}, \frac{2}{v}} \cdot\{(1,0,0) ;(0,0,-1) ;(0,-1,0) \mid \text { respectively }\} .
\end{aligned}
$$

We now re-demonstrate that the above $\left\{\psi_{\mathrm{A}}, \psi_{\mathrm{B}}\right\}$ solve the Dirac equation.

At $t=1 / v$,

$$
\sigma_{1} i \nabla\left(\psi_{A}, \psi_{B}\right)=\left(\begin{array}{ll}
0 & 1 \\
1 & 0
\end{array}\right)_{(x, y)}\left(\begin{array}{ll}
0 & 1 \\
1 & 0
\end{array}\right)_{(x, y)} \frac{k}{2 \alpha} \cdot\left(\psi_{A}, \psi_{B}\right)=\frac{k}{2 \alpha} \cdot\left(\psi_{A}, \psi_{B}\right)
$$

at $t=1.5 / v$,

$$
\sigma_{2} i \nabla\left(\psi_{A}, \psi_{B}\right)=\left(\begin{array}{cc}
1 & 0 \\
0 & -1
\end{array}\right)_{(x, z)}\left(\begin{array}{cc}
1 & 0 \\
0 & -1
\end{array}\right)_{(x, z)} \frac{k}{2 \alpha} \cdot\left(\psi_{A}, \psi_{B}\right)=\frac{k}{2 \alpha} \cdot\left(\psi_{A}, \psi_{B}\right)
$$

at $t=2 / v$,

$$
\begin{aligned}
& \sigma_{3} i \nabla\left(\psi_{A}, \psi_{B}\right)=\left(\begin{array}{cc}
0 & -i \\
i & 0
\end{array}\right)_{(x, y)}\left(\begin{array}{cc}
0 & 0 \\
0 & -1 \\
1 & 0
\end{array}\right)_{(x, y, z)} \frac{k}{2 \alpha} \cdot\left(\psi_{A}, \psi_{B}\right) \\
& \equiv\left(\begin{array}{cc}
0 & -i \\
i & 0
\end{array}\right)_{(x, y)}\left(\begin{array}{cc}
0 & -i \\
i & 0
\end{array}\right)_{(x, y)} \frac{k}{2 \alpha} \cdot\left(\psi_{A}, \psi_{B}\right)=\frac{k}{2 \alpha} \cdot\left(\psi_{A}, \psi_{B}\right) .
\end{aligned}
$$

Thus, $\forall j=1,2,3$

$$
c \sigma_{j} P_{(x, y, z)}\left(\psi_{A}, \psi_{B}\right) \equiv-c \sigma_{j} i \hbar \nabla\left(\psi_{A}, \psi_{B}\right)=-\frac{c \hbar k}{2 \alpha}\left(\psi_{A}, \psi_{B}\right)=-\frac{p c}{\alpha}\left(\psi_{A}, \psi_{B}\right)
$$

setting 


$$
\alpha=\frac{p c}{E-m_{0} c^{2}} \in(0,1),
$$

avalue that depends on the reference frame, we have

$$
c \sigma_{j} P_{(x, y, z)}\left(\psi_{A}, \psi_{B}\right)=-\left(E-m_{0} c^{2}\right)\left(\psi_{A}, \psi_{B}\right) .
$$

Now

$$
P_{t} \psi \equiv i \hbar \frac{\partial \psi}{\partial t}=\frac{\hbar \omega}{2} \cdot \psi=E \psi
$$

so that

$$
\left(P_{t}+c \sigma_{j} P_{(x, y, z)}\right)\left(\psi_{A}, \psi_{B}\right)=E \cdot\left(\psi_{A}, \psi_{B}\right)-\left(E-m_{0} c^{2}\right) \cdot\left(\psi_{A}, \psi_{B}\right)=m_{0} c^{2} \cdot\left(\psi_{A}, \psi_{B}\right) .
$$

In this way, we solved the Dirac equation and we have the wave motion for a free electron to be

$$
\begin{aligned}
& \Lambda_{A}\left(t=\frac{1}{v}, \frac{1.5}{v}, \frac{2}{v}, \frac{2.5}{v}, \frac{3}{v}\right)=(W, N, E, T, W) \text { or } \\
& \Lambda_{B}\left(t=\frac{1}{v}, \frac{1.5}{v}, \frac{2}{v}, \frac{2.5}{v}, \frac{3}{v}\right)=(T, E, B, S, T) .
\end{aligned}
$$

Here we note that $\Lambda_{\mathrm{B}}$ is actually redundant since $\Lambda_{\mathrm{A}}$ and $\Lambda_{\mathrm{B}}$ are the same physical object viewed by two different frames. That is, consider first rotating the $x$-z plane around the $y$-axis clockwise by 90 degrees; then $R_{(x, z)}(W, T, E)$ $=(T, E, B)$. Next, rotating the $x-y$ plane around the $z$-axis clockwise by 90 degrees, we have $R_{(x, y)}(N, E)=(E, S)$ and thus

$$
\left(R_{(x, y)} \circ R_{(x, z)}\right)(W, N, E, T, W)=(T, E, B, S, T) .
$$

Formally,

$$
\begin{aligned}
& R_{(x, y)}=\left(\begin{array}{ccc}
0 & 1 & 0 \\
-1 & 0 & 0 \\
0 & 0 & 1
\end{array}\right), R_{(x, z)}=\left(\begin{array}{ccc}
0 & 0 & 1 \\
0 & 1 & 0 \\
-1 & 0 & 0
\end{array}\right), \text { and } \\
& R_{(x, y)} \circ R_{(x, z)}=\left(\begin{array}{ccc}
0 & 1 & 0 \\
0 & 0 & -1 \\
-1 & 0 & 0
\end{array}\right), \text { mapping } \\
& \left(\begin{array}{lll}
0 & 1 & 0 \\
1 & 0 & 0 \\
0 & 0 & 1
\end{array}\right) \text { to }\left(\begin{array}{ccc}
1 & 0 & 0 \\
0 & 0 & -1 \\
0 & -1 & 0
\end{array}\right), \text { i.e., }(N, E, T) \mapsto(E, B, S) .
\end{aligned}
$$

As such, $\Lambda_{A}(t)$ alone suffices to describe the motion of a free electron-wave, a path that is the union of two perpendicularly connected semi-circles,

$$
S_{(x, y \geq 0)}^{1} \cup S_{(x, z \geq 0)}^{1} .
$$

In passing, we note here that the motion $\Lambda_{A}(t)$ explains why it takes $180 \times 4$ degrees to restore an electron-wave: the first sequence of $W \rightarrow N \rightarrow E \rightarrow T \rightarrow W$ turns the $z$-axis upside down to $-z$, so that the second sequence of $W \rightarrow N \rightarrow E \rightarrow T \rightarrow W$ restores $-z$ to $z$ (cf. Ahrens et al., 2012, forspin-flip; Karlovets, 2012, for "twisted electrons").

We also note that the sequence of $\sigma_{1} \rightarrow \sigma_{2} \rightarrow \sigma_{3}$ defines one spin direction and its reverse $\sigma_{3} \rightarrow \sigma_{2} \rightarrow \sigma_{1}$ defines the opposite spin direction, $W \rightarrow T \rightarrow E \rightarrow N \rightarrow W$ (cf. the CPT theorem).

To sum up, there exists exactly one path $S_{(x, y \geq 0)}^{1} \cup S_{(x, z \geq 0)}^{1}$ with two alternative spin directions for an electron-wave. We now project this path onto a $y-z$ plane, with $(x, y, z)=(0,1,0) \equiv N$ identified as the 0 -o'clockposition and $(x, y, z)=(0,0,1) \equiv T$ identified as the 3-o'clock position. Then we have the following 
representation,

$$
S_{(x, y \geq 0)}^{1} \cup S_{(x, z \geq 0)}^{1}=(0,3),
$$

denoting a field energy outflow commencing at $\boldsymbol{W}$ traveling through $0 \equiv N$ stopping at $\boldsymbol{E}$ and then changing the plane to continue to travel through $3 \equiv T$ back to $\boldsymbol{W}$. As such, $(3,0) \equiv[W \rightarrow T \rightarrow E \rightarrow N \rightarrow W]$ represents an electron-wave of the opposite spin.

Since $\left\{-\sigma_{1},-\sigma_{2},-\sigma_{3}\right\}$ result in a positron by the Dirac equation, we have

$$
\begin{aligned}
& (6,9) \equiv[E \rightarrow S \rightarrow W \rightarrow B \rightarrow E] \text { and } \\
& (9,6) \equiv[E \rightarrow B \rightarrow W \rightarrow S \rightarrow E]
\end{aligned}
$$

as the two alternative spins of a positron-wave. In this way, $(0,3) \cup(6,9)$ can be recombined into $(0,6) \cup(3,9)$, i.e.,

$$
[W \rightarrow N \rightarrow E \rightarrow S \rightarrow W] \cup[E \rightarrow T \rightarrow W \rightarrow B \rightarrow E]=S_{(x, y)}^{1} \cup S_{(x, z)}^{1},
$$

as two photon-waves.

If we generalize the Pauli matrix

$$
\sigma_{3}=\left(\begin{array}{cc}
0 & -i \\
i & 0
\end{array}\right)_{(x, y)} \text { to } \quad \tilde{\sigma}_{3}=\left(\begin{array}{cc}
0 & a-b i \\
a+b i & 0
\end{array}\right)_{(x, y)}, \quad \text { with } a^{2}+b^{2}=1
$$

then $\tilde{\sigma}_{3}=I$ and the Dirac equation is still satisfied; in particular, $\frac{\sqrt{3}}{2} \pm \frac{1}{2} i$ and $\frac{1}{2} \pm \frac{\sqrt{3}}{2} i$ would respectively represent field energy flows

$$
\begin{aligned}
& {\left[W \rightarrow N \rightarrow E \rightarrow T_{N N} \rightarrow W\right] \equiv(0,1) \text { and }} \\
& {\left[W \rightarrow N \rightarrow E \rightarrow T_{T N} \rightarrow W\right] \equiv(0,2),}
\end{aligned}
$$

alongwith their alternative spins $(1,0)$ and $(2,0)$. To pursue further analysis, we need set up the following definition and notation.

Definition set $0 \equiv S_{(x, y \geq 0)}^{1}$. As projected onto the $y-z$ plane: $1 \equiv(0$ rotated clockwise by 30 degrees $)$, $2 \equiv(0$ rotated clockwise by 60 degrees $), \quad$ and $3 \equiv(0$ rotated clockwise by 90 degrees $) . \quad \forall\{a, b\} \subset\{0,1,2,3\}$ define

\section{$(a, b):=(a$ field energy outflow commencing at $W$ along a to $E$,} followed by its inflow from $E$ along $b$ back to $W$ ).

Since electron $e^{-}$has two spin directions $(0,3) \equiv W \stackrel{0}{\longrightarrow} E \stackrel{3}{\longrightarrow} W$ and $(3,0) \equiv W \stackrel{3}{\longrightarrow} E \stackrel{0}{\longrightarrow} W$, we shall distinguish between these two distinct spin directions by defining spin $=-1 / 2$ for any motions $W \stackrel{a}{\longrightarrow} E \stackrel{b}{\longrightarrow} W$ such that $b-a \in\{0,1,2,3\}$ and spin $=1 / 2$ for any motions such that $b-a \in\{-1,-2,-3\}$.

In view of the rotational invariance of the angle $\angle(a, b)=b-a$, we have the equivalence relation

$$
(a, b) \simeq(a+k, b+k) \forall k \in Z .
$$

Recalling (from Equation (2.26)) that positron $e^{+}$has the motions $(6,9) \equiv E \stackrel{6}{\longrightarrow} W \stackrel{9}{\longrightarrow} E$ or $(9,6)=E \stackrel{9}{\longrightarrow} W \stackrel{6}{\longrightarrow} E$, we observe that the reference point for anti-particles becomes $E \equiv-W$ and $(c, d)_{E}=(a+6, b+6)_{-W}$; i.e., $\left(-\sigma_{j=1,2,3}\right)$ from the Dirac equation for $e^{+}$is equivalent to $\left(\sigma_{j=1,2,3}\right)$ by the coordinate system $\{(-x,-y,-z)\}$ (cf. the CPT theorem). To maintain the clear identity of anti-particles, we will hereafter use the sharp brackets $\langle c, d\rangle_{E}$ to represent anti-particles, and $(a, b)_{W}$ for particles. Thus, for example, we have the following four distinct particles: $e^{-}{ }_{(-1 / 2)}=(0,3)_{W}, e_{(1 / 2)}^{-}=(3,0)_{W}, e^{+}{ }_{(-1 / 2)}=<0,3>_{E}$, and $e_{(1 / 2)}^{+}=<3,0>_{E}$. 
To prepare for the ensuing analysis of particle collisions, we will use the symbol $\boxplus$ for field superpositions, and we note that the mixing angles among the colliding fields determine the outcomes, a well-observed fact (cf. Alvarez et al., 2013; Tayduganov et al., 2012). As a demonstration, consider the pair-annihilation of $e^{-}$and $e^{+}$; one would need rotating $\langle 0,3\rangle_{E}$ by an angle of 180 degrees to $\left.<6,9\right\rangle_{E}$ in order to have

$$
\begin{aligned}
& (0,3)_{W} \boxplus\langle 6,9\rangle_{E}=W \stackrel{0}{\longrightarrow} E \stackrel{6}{\longrightarrow} W \stackrel{9}{\longrightarrow} E \stackrel{3}{\longrightarrow} W \\
& =[0,6]_{W} \boxplus[9,3]_{W}=S_{(x, y),(-1)}^{1}+S_{(x, z),(+1)}^{1},
\end{aligned}
$$

to result in two photon-waves (noting here that the angle of 180 degrees of separation refers to the final state of field superposition, which is not to be identified with the mixing angle responsible for arriving at this state). Here and after we will use the square brackets for particle-waves resulting from a combination of particles and anti-particles.

Since neutrinos $v$ have the well-known parity asymmetry of possessing only one spin direction, we reason that their wave motions must be $(0,0)_{W}=W \stackrel{0}{\longrightarrow} E \stackrel{0}{\longrightarrow} W$. This in turn suggests that the electric charge of $(a, b)_{W}$ or $\langle a, b\rangle_{E}$ equals $\pm(1 / 3)(b-a)$, so that quarks $\{d, s, b\}$ have representations $(0, \pm 1)_{W}$ and $\{u, c, t\}$ have representations $(0, \pm 2)_{W}$ (cf. Corben, 1961, for an earlier struggle to make sense of "intrinsic spin," and most recently Aidala et al., 2013, for a critical re-examination of this concept).

As an illustration, we analyze the neutron decay as follows:

$$
\begin{aligned}
& n=\{u, d, d\}=\{u, u, d\} \boxplus\{d-u\} \\
& =\left\{(2,0)_{W} \boxplus(4,2)_{W} \boxplus(3,4)_{W}\right\} \boxplus\left\{(0,1)_{W} \boxplus(1,3)_{W}\right\} \\
& =(3,0)_{W} \boxplus(0,3)_{W} \boxplus\left(1_{\text {in }}, 1_{\text {out }}\right)_{W} \\
& =p \boxplus e^{-} \boxplus\langle 1,1\rangle_{E}=p \boxplus e^{-} \boxplus \bar{v}_{e},
\end{aligned}
$$

where the embodiment of $\{u \boxplus(-u)\}$ in neutron $n$ results in $\left(1_{\text {in }}, 1_{\text {out }}\right)_{W} \equiv<1,1>_{E}$, i.e., the breaking away of $\bar{v}_{e}$. Here we need to make some additional remark about neutrinos. In the above $\beta$ decay we had

$$
\begin{aligned}
& (0,1)_{W} \boxplus(1,3)_{W}=W \stackrel{0}{\longrightarrow} E \stackrel{1}{\longrightarrow} W \stackrel{1}{\longrightarrow} E \stackrel{3}{\longrightarrow} W \\
& =W \stackrel{0}{\longrightarrow} E \stackrel{1}{\longrightarrow} W \stackrel{1}{\longrightarrow} E ; \stackrel{3}{\longrightarrow} W \\
& =\{W \stackrel{0}{\longrightarrow} E \stackrel{3}{\longrightarrow} W\} \boxplus\{E \stackrel{1}{\longrightarrow} W \stackrel{1}{\longrightarrow} E\} \\
& =(0,3)_{W} \boxplus\langle 1,1\rangle_{E}=e^{-} \boxplus \bar{v}_{e} .
\end{aligned}
$$

One can see that in the above $\beta$ decay it definitely requires an anti-neutrino $\bar{v}_{e}$ to effect the process. However, once $\bar{\nu}_{e}$ becomes a free particle, its wave motion progresses as

$$
E \stackrel{1}{\longrightarrow} W \stackrel{1}{\longrightarrow} E \stackrel{1}{\longrightarrow} W \stackrel{1}{\longrightarrow} E \stackrel{1}{\longrightarrow} W \cdots
$$

i.e., $(1,1)_{W} \equiv<1,1>_{E}$, a Majorana fermion (for the recent intensive global research on neutrinos, see, e.g., Zvyagin, 2013; Singh et al., 2006).

We now present some additional examples as below.

$$
\Lambda=\{u, d, s\}=(2,0)_{W} \boxplus(0,1)_{W} \boxplus(1,2)_{W},
$$

with electric charge $=0$ and $\operatorname{spin}=1 / 2-1 / 2-1 / 2=-1 / 2$ (recall that a particle can take either $(a, b)_{W}$ or $(b, a)_{W}$ so that here the spin $\left.= \pm 1 / 2\right)$.

$$
\Omega-=\{s, s, s\}=(0,1)_{W} \boxplus(1,2)_{W} \boxplus(2,3)_{W},
$$

with electric charge $=-1$ and $\operatorname{spin}=-1 / 2-1 / 2-1 / 2=-3 / 2$.

$$
\pi+=\{u, \bar{d}\}=(1,3)_{W} \boxplus<6,7>_{E}=(1,3)_{W} \boxplus<0,1>_{E}
$$




$$
=W \stackrel{1}{\longrightarrow} E \stackrel{0}{\longrightarrow} W \stackrel{1}{\longrightarrow} E \stackrel{3}{\longrightarrow} W=[1,0]_{W} \boxplus[1,3]_{W},
$$

with electric charge $=1\left(=\frac{1}{3} \times(3-0)\right.$ from the spanned angle $\left.\angle(0,3)\right)$ and spin $=1 / 2-1 / 2=0$.

$$
\begin{gathered}
\left.\rho+=\{u, \bar{d}\}(\text { same as in } \pi+)=(1,3)_{W} \boxplus<7,6>_{E} \text { (cf. } \pi+\text { here) }=(1,3)_{W}+<1,0\right\rangle_{E} \\
=W \stackrel{1}{\longrightarrow} E \stackrel{1}{\longrightarrow} W \stackrel{0}{\longrightarrow} E \stackrel{3}{\longrightarrow} W=[1,1]_{W} \boxplus[0,3]_{W},
\end{gathered}
$$

with electric charge $=1$ and spin $=-1 / 2-1 / 2=-1$ (cf. $\pi+$ here) .

$$
\begin{gathered}
K-=\{s, \bar{u}\}=(2,3)_{W} \boxplus<6,8>_{E}=(2,3)_{W} \boxplus<0,2>_{E} \\
=W \stackrel{2}{\longrightarrow} E \stackrel{0}{\longrightarrow} W \stackrel{2}{\longrightarrow} E \stackrel{3}{\longrightarrow} W=[2,0]_{W} \boxplus[2,3]_{W},
\end{gathered}
$$

with electric charge $=-1$ and spin $=1 / 2-1 / 2=0$.

$$
\begin{aligned}
B^{0}=\{d, \bar{b}\}=(0,1)_{W} \boxplus<6,7>_{E} & =W \stackrel{0}{\longrightarrow} E \stackrel{6}{\longrightarrow} W \stackrel{7}{\longrightarrow} E \stackrel{1}{\longrightarrow} W=[0,6]_{W} \boxplus[7,1]_{W} \\
& =S_{(x, y),(-1)}^{1} \boxplus S_{(x, y, z),(+1)}^{1},
\end{aligned}
$$

with electric charge $=0$ and spin $=-1+1=0$.

$$
\begin{gathered}
J / \psi=\{c, \bar{c}\}=(0,2)_{W} \boxplus<6,8>_{E}=W \stackrel{0}{\longrightarrow} E \stackrel{6}{\longrightarrow} W \stackrel{8}{\longrightarrow} E \stackrel{2}{\longrightarrow} W=[0,6]_{W} \boxplus[8,2]_{W} \\
=S_{(x, y),(-1)}^{1} \boxplus S_{(x, y, z),(+1)}^{1},
\end{gathered}
$$

with electric charge $=0$ and spin $=-1+1=0$.

\section{Summary Remark}

By providing all fermions and bosons with the geometrices of their wave motions, we have amplified the quark model for an explanation of the hundreds' varieties of nuclear particles, all via different superpositions of the electromagnetic fields in the cosmic black hole $\boldsymbol{B}$ contained in the wave universe $\boldsymbol{M}^{[2]}$ (see Light, 2013, Equation (1.1)). To be sure, our mathematical framework permits non-quark particles by admitting additional angles between the two semi-circles mentioned above, such as 45 degrees, in which case, the electric charge $= \pm 1 / 2$ (cf. Chatrchyan et al., 2013, which, however, still remained in the considerations of fractions with the common denominator of three).

The Standard Model, built upon quantizing all energies into particles, serves only as a partial presentation of the reality. 'The tyranny of the standard model, which has plagued particle physics for decades, has now reached cosmology as well,' remarked MIT physicist Tegmark (quotation originally by Science reporter Cho, 2013, who wrote in the News\& Analysis column of the AAAS magazine: “... standard model accounts for everything discovered with atom smashers-including the recently spotted Higgs boson-but leaves bigger conceptual questions untouched."). As is well-known, theoretically the Standard Model has failed to account for gravity. Experimentally enigmatic discoveries under the title "new physics" have constantly been made (cf. e.g., a most recent finding by Ablikim et al., 2013). In our analytical framework, potentially unlimited superpositions of the continuous energy waves in $\boldsymbol{B}$ of $\boldsymbol{M}^{[2]}$ are the sources of the discrete particles, and therefore it is not surprising that new particles keep being found. Inconclusion, for a complete description of the Universe we propose the setup of two independent sets of Einstein Field Equations that are unified as our combined spacetimefour-manifold $\boldsymbol{M}^{[3]}$ containing particle-waves in $\{(t+t i, x+y i, y+z i, z+x i)\}$ with their motions specified in this paper. 


\section{References}

Ablikim, M., Achasov, M. N., Ai, X. C., Albayrak, O., Ambrose, D. J., An, F. F., ... Zou, J. H. (2013). Observation of a charged charmoniumlikestructure in $\mathrm{e}+\mathrm{e}-\rightarrow \pi+\pi-\mathrm{J} / \psi$ at $\sqrt{\mathrm{s}}_{\mathrm{s}}=4.26 \quad \mathrm{GeV}$. Physical Review Letters, 110, 252001 (1-7). http://dx.doi.org/10.1103/PhysRevLett.110.252001

Ahrens, S., Bauke, H., Keitel, C. H., \& Muller, C. (2012). Spin dynamics in the Kapitza-Dirac effect. Physical Review Letters, 109, 043601 (1-5). http://dx.doi.org/10.1103/PhysRevLett.109.043601

Aidala, C. A., Bass, S. D., Hasch, D., \& Mallot, G. K. (2013). The spin structure of the nucleon. Reviews of Modern Physics, 85, 655-691. http://dx.doi.org/10.1103/RevModPhys.85.655

Alvarez, E., Vietto, J. I. S., \& Szynkman, A. (2013). Top-antitop resonance searches beyond $1 \mathrm{TeV}$. Physical Review D, 87, 054015 (1-11). http://dx.doi.org/10.1103/PhysRevD.87.054015

Barut, A. O., \& Bracken, A. J. (1981). Zitterbewegung and the internal geometry of the electron. Physical Review D, 23, 2454-2463. http://dx.doi.org/10.1103/PhysRevD.23.2454

Chatrchyan, S., Khachatryan, V., Sirunyan, A. M., Tumasyan, A., Adam, W., Aguilo, E., ... Swanson, J. (2013). Search for fractionally charged particles in pp collisions at $\sqrt{s}_{\mathbf{s}}=7$ TeV. Physical Review D, 87, 092008 (1-17). http://dx.doi.org/10.1103/PhysRevD.87.092008

Cho, A. (2013). Universe's high-def baby picture confirms standard theory. Science, 339, 1513. http://dx.doi.org/10.1126/science.339.6127.1513

Corben, H. C. (1961). Spin in classical and quantum theory. Physical Review, 121, 1833-1839. http://dx.doi.org/10.1103/PhysRev.121.1833

Karlovets, D. V. (2012). Electron with orbital angular momentum in a strong laser wave. Physical Review A, 86, 062102 (1-13). http://dx.doi.org/10.1103/PhysRevA.86.062102

Le'vay, P., Saniga, M., \&Vrana, P. (2008).Three-qubit operators, the split Cayley hexagon of order two, and black holes. Physical Review D, 78, 124022 (1-16). http://dx.doi.org/10.1103/PhysRevD.78.124022

Light, G. L. (2013).A detailed analysis of the quantum relativistic motion of an electron. Applied Physics Research, 5(1), 7-18. http://dx.doi.org/10.5539/apr.v5n1p7

Rafanelli, K., \& Schiller, R. (1964). Classical motions of spin-1/2 particles. Physical Review, 135, B279-B281. http://dx.doi.org/10.1103/PhysRev.135.B279

Singh, D., Mobed, N., \& Papini, G. (2006). Can gravity distinguish between Dirac and Majorana neutrinos? Physical Review Letters, 97, 041101 (1-4). http://dx.doi.org/10.1103/PhysRevLett.97.041101

Taub, A. H. (1949). A special method for solving the Dirac equations. Reviews of Modern Physics, 21, 388-392. http://dx.doi.org/10.1103/RevModPhys.21.388

Tayduganov, A., Kou, E., \& Le Yaouanc, A. (2012). Strong decays of $\mathrm{K}_{1}$ resonances. Physical Review D, 85, 074011(1-28). http://dx.doi.org/10.1103/PhysRevD.85.074011

Zvyagin, A. A. (2013). Possibility of direct observation of edge Majorana modes in quantum chains. Physical Review Letters, 110, 217207(1-5). http://dx.doi.org/10.1103/PhysRevLett.110.217207

\section{Copyrights}

Copyright for this article is retained by the author(s), with first publication rights granted to the journal.

This is an open-access article distributed under the terms and conditions of the Creative Commons Attribution license (http://creativecommons.org/licenses/by/3.0/). 